\title{
Crosstalk Analysis of Asynchronous Optical Chip Interconnects with Direct Detection
}

\author{
Tri T. Ha, Fellow, IEEE, and Rena M. Loesch
}

\begin{abstract}
We provide a crosstalk analysis of optical interconnects via single-mode waveguides with asynchronous transmission. Our crosstalk model is general and can be used for any type of waveguide network. The analysis takes into account the coupling-induced crosstalks between adjacent waveguides, the laser linewidth, the shot noise and the dark current generated by the photodiode, and the postdetection thermal noise. $A$ comparison to synchronous transmission is also provided.
\end{abstract}

\section{INTRODUCTION}

$\mathbf{W}$ E consider the problem of crosstalk impact in an optical interconnect system using a single-mode waveguide network [1]-[7]. An optical interconnect consists of two or more terminal nodes which are interconnected by a single optical path or a high-density parallel optical network which preserves the parallel nature of the data generated at the nodes. Such an interconnect would consist of chip-to-chip or board-to-board interconnections [8]. The use of optical singlemode waveguides provides a potential of integrating the entire system of transmitter and receiver on the same substrate [9].

We consider a mathematical approach that includes more than two interfering channels. The modulation format used in our analysis is on-off keying (OOK) with direct detection. Our emphasis will be on the impact of coupling-induced crosstalk between adjacent waveguides in a waveguide network. The additional parameters that determine the performance of the interconnect system are the laser linewidth, the shot noise and the dark current generated by the photodiode, and the postdetection thermal noise. To be general, we consider the receiver to be an integrate-and-dump filter with integration time $T$, where $T$ is the bit time. Also, we denote $N_{0}$ as the spectral density of the postdetection thermal noise current. The spectral density $N_{0}$ can be easily computed for a given low noise amplifier type given the effective noise temperature and the matched resistance load.

The optical interconnect system is given in Fig. 1 for OOK with direct detection. The waveguide network consists of many single-mode waveguides. It can be a planar array with uniform waveguide separation or any other structure. The envelope of the output lightwave of a given waveguide is detected by the photodiode, which also generates shot noise and dark current. The output current of the photodiode is further corrupted by the amplifier thermal noise current. The total signal current plus crosstalk, shot noise, dark current, and thermal noise

Manuscript received December 9, 1993; revised September 6, 1994.

The authors are in the Department of Electrical and Computer Engineering, Naval Postgraduate School, Monterey, CA 93943-5121 USA

IEEE Log Number 9405767. current is integrated over one bit time $T$, and the resulting bit energy at the end of each integration time is determined by the slicer to be either bit one or bit zero. We assume that the waveguide bandwidth is much larger than the bit rate and the signal spectrum.

As in [8], we will consider three cases of laser sources. In case one, each channel operates with an independent laser source. In case two, all laser sources have the same mean wavelength but with different phase noise processes. In case three, all channels share the same master source with possibly the exceptions of the initial phases.

This paper is organized into four sections. Section II provides the theoretical framework for the performance analysis when the bit transmission is assumed to be asynchronous; that is, bit streams in all channels are not time-aligned. Section III discusses the numerical results, and a summary of the study appears in Section IV.

\section{ANALYSIS}

For mathematical convenience, we adopt the complex envelope notation of a real signal. Thus, for a given transmitted $i$ th bit $b_{0}$ of a given channel 0 whose laser phase noise process is $\theta_{0}(t)$, the signal at the input of the photodiode is designated as

$$
\begin{aligned}
s(t)= & \frac{A}{\sqrt{2}} b_{0} e^{j \theta_{0}(t)}[u(t)-u(t-T)] \\
& +\sum_{k=1}^{M} \frac{C_{k} A}{\sqrt{2}} b_{k,-1} e^{j\left[\theta_{k}(t)+\omega_{k} t+\phi_{k},-1\right]} \\
& \cdot\left[u(t)-u\left(t-\tau_{k}\right)\right] \\
& +\sum_{k=1}^{M} \frac{C_{k} A}{\sqrt{2}} b_{k, 0} e^{j\left[\theta_{k}(t)+\omega_{k} t+\phi_{k}, 0\right]} \\
& \cdot\left[u\left(t-\tau_{k}\right)-u(t-T)\right]
\end{aligned}
$$

where $A$ is the OOK signal amplitude, $b_{k,-1}$ and $b_{k, 0}$ are the previous bit and the present bit in channel $k$ relative to bit $b_{0}, \theta_{k}(t)$ is the laser phase noise process, $\omega_{k}$ and $\phi_{k,-1}$ and $\phi_{k, 0}$ are the frequency spacing and the initial phase differences between channels $k$ and 0 , respectively. Also, $C_{k}$ represents the coupling from channel $k$ to channel 0 . The parameter $\tau_{k}$ represents the uniformly distributed random delay between bit $b_{0}$ and $b_{k, 0}$. The function $u(t)$ denotes the unit step function. The summation term in (1) thus represents the crosstalk from $M$ adjacent channels into channel 0 . In (1), we have assumed that all waveguides have the same attenuation. For the case of $M=2$ and synchronous transmission, that is, 


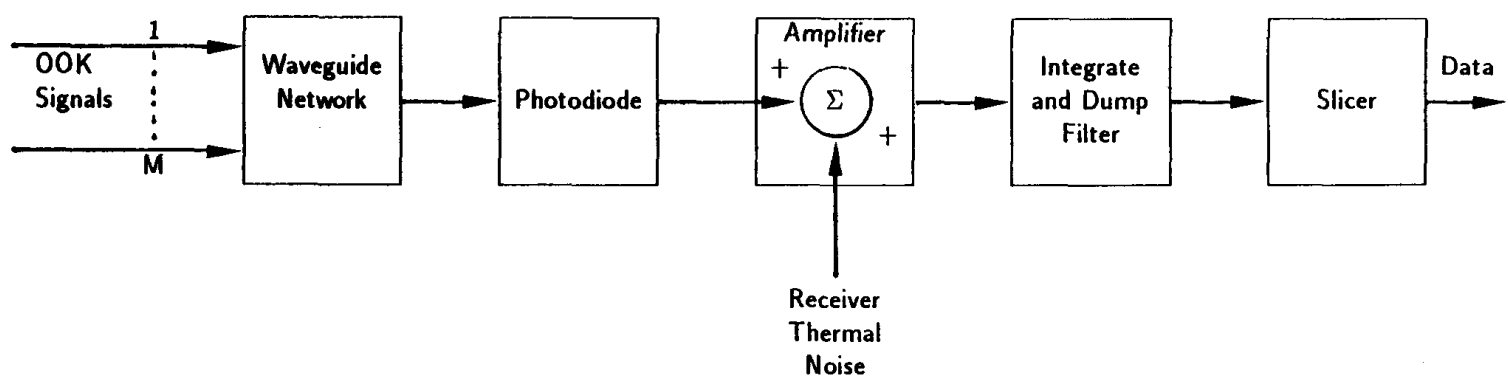

(a)

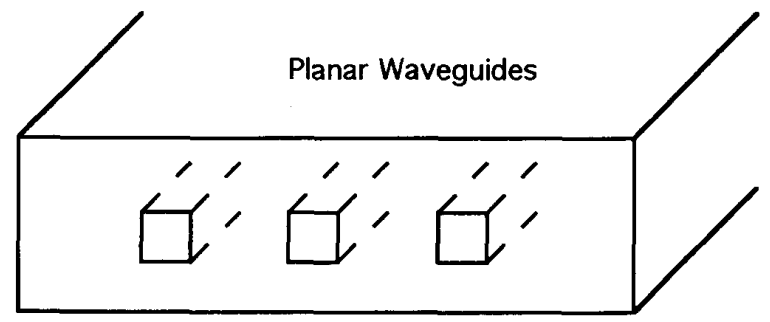

(b)

Fig. 1. (a) A block diagram of an optical chip interconnect. (b) A planar waveguide network.

only the two nearest adjacent channels are considered, then (1) reduces to the result in [8, eq. (2.4)]. In this case, $C_{k}=$ $B / \sqrt{1-2 B^{2}}, k=1,2$ where $B$ is the total power coupled from a waveguide into its adjacent waveguide. In practice, $b_{k,-1}$ and $b_{k, 0}, k=0,1, \cdots, M$ are not necessarily equal to zero or one. Let $r_{k}, k=0,1, \cdots, M$ be the extinction ratio of the laser of channel $k$ defined as the ratio of the transmitted power of the logical zero to that of a logical one. Then $b_{k, 0}=b_{k,-1}=\sqrt{r_{k} /\left(1+r_{k}\right)}$ for logical zero and $b_{k, 0}=b_{k,-1}=\sqrt{1 /\left(1+r_{k}\right)}$ for logical one. Hereafter, we assume $r_{k}$ 's are identical for all channels.

Let $R=n_{e} q / h f$ be the photodiode responsitivity [10]-[12], where $n_{e} \leq 1$ is the quantum efficiency, $q$ is the electron charge $\left(1.6 \times 10^{-19} \mathrm{C}\right), h$ is Planck's constant $\left(6.626 \times 10^{-34} \mathrm{~J}\right.$. $\mathrm{s})$, and $f$ is the frequency. Then the output of the photodiode is $R|s(t)|^{2}+w_{s}(t)+w_{d k}(t)$, where $w_{s}(t)$ is the shot noise current generated by the photodiode, and $w_{d k}(t)$ is the dark current noise. The output of the photodiode plus the postdetection thermal noise current $n(t)$ is integrated by the integrate-anddump filter with the normalization constant $R$ to provide the decision variable $Y$ as follows:

$$
\begin{aligned}
Y= & \frac{1}{R} \int_{0}^{T} R|s(t)|^{2} d t+\frac{1}{R} \int_{0}^{T} w_{s}(t) d t+\frac{1}{R} \int_{0}^{T} w_{d k}(t) d t \\
& +\frac{1}{R} \int_{0}^{T} n(t) d t \\
= & X+W_{s}+W_{d k}+N
\end{aligned}
$$

where

$$
\begin{aligned}
X & =\int_{0}^{T}|s(t)|^{2} d t \\
W_{s} & =\frac{1}{R} \int_{0}^{T} w_{s}(t) d t
\end{aligned}
$$

$$
\begin{aligned}
W_{d k} & =\frac{1}{R} \int_{0}^{T} w_{d k}(t) d t \\
N & =\frac{1}{R} \int_{0}^{T} n(t) d t .
\end{aligned}
$$

Since $n(t)$ is a zero mean Gaussian process with spectral density $N_{0}$, the Gaussian random variable $N$ also has zero mean and its variance $\sigma_{N}^{2}$ is given by

$$
\sigma_{N}^{2}=\frac{T N_{0}}{R^{2}}
$$

On the other hand, the shot noise current $w_{s}(t)$ is a nonstationary process since the envelope of the signal at the input of the photodiode, namely, $|s(t)|$ is timedependent. For tractable analysis, we can model the shot noise $w_{s}(t)$ as a zero-mean wide-sense stationary Gaussian process whose spectral density $W_{0}(\boldsymbol{b})$ given a bit pattern $\boldsymbol{b}=\left(b_{0}, b_{1,-1}, \cdots, b_{M,-1}, b_{1,0}, \cdots, b_{M, 0}\right)$ is proportional to the conditional mean of the squared envelope of the input signal [13]. In other words, we approximate $|s(t)|^{2}$ by $E\left\{|s(t)|^{2} \mid \boldsymbol{b}\right\}$ over a bit time $T$. Based on this approximation, the shot noise current spectral density can be obtained as follows:

$$
\begin{aligned}
W_{0}(\boldsymbol{b}) & =q R E\left\{|s(t)|^{2} \mid \boldsymbol{b}\right\} \\
& =\frac{1}{2} q R A^{2}\left(b_{0}^{2}+\frac{1}{2} \sum_{k=1}^{M} C_{k}^{2} b_{k,-1}^{2}+\frac{1}{2} \sum_{k=1}^{M} C_{k}^{2} b_{k, 0}^{2}\right) .
\end{aligned}
$$

From (2c) and (4), the conditional variance of $W_{s}$ given a bit pattern $\boldsymbol{b}$ is

$$
\sigma_{W_{s}}^{2}(\boldsymbol{b})=\frac{T W_{0}(\boldsymbol{b})}{R^{2}}
$$


The dark current noise spectral density function is $q I_{d k}$, where $I_{d k}$ is the dark current. The variance of the dark current noise is $\sigma_{W_{d k}}^{2}=T q I_{d k} / R^{2}$. The random variable $X$ in (2b) consists of the signal term, the signal-crosstalk terms, and the crosstalk-crosstalk terms.

The statistics of the crosstalk terms are extremely difficult to obtain (if possible). Therefore, for tractable analysis, we model $Y$ as a Gaussian random variable. Such a Gaussian approximation has also been used in [8] with a different mathematical approach for the synchronous transmission case. Gaussian approximations are commonly used to obtain the bit error probability for lightwave systems when the exact statistics of the decision variable cannot be analytically obtained [14]-[16]. The Gaussian approximation is conservative and normally overestimates the bit error probability for small laser linewidths or channel spacings. For the Gaussian approximation to be accurate, the laser linewidth or the channel spacing must be at least twice the bit rate. From (2b), the mean of $X$ conditional on a given bit pattern $\boldsymbol{b}$ is given by

$$
\begin{aligned}
\bar{X}(\boldsymbol{b})= & E\{X \mid \boldsymbol{b}\}=\frac{1}{2} A^{2} T b_{0}^{2}+\frac{1}{4} \sum_{k=1}^{M} C_{k}^{2} A^{2} T b_{k,-1}^{2} \\
& +\frac{1}{4} \sum_{k=1}^{M} C_{k}^{2} A^{2} T b_{k, 0}^{2} .
\end{aligned}
$$

From (6) and (A5) of the Appendix, the conditional variance $\sigma_{X}^{2}(\boldsymbol{b})$ of $X$ can be calculated as follows:

$$
\begin{aligned}
\sigma_{X}^{2}(\boldsymbol{b})= & \sum_{k=1}^{M} C_{k}^{2} A^{4} T^{2} b_{0}^{2}\left(b_{k,-1}^{2}+b_{k, 0}^{2}\right)\left\{\frac{\pi \nu}{4 \pi^{2}\left(\nu^{2}+\delta_{k}^{2}\right)}\right. \\
& -\frac{1}{64 \pi^{6}\left(\nu^{2}+\delta_{k}^{2}\right)^{3}}\left(8 \pi ^ { 2 } \nu \delta _ { k } \left[2 \pi \delta_{k}\right.\right. \\
& \left.-e^{-2 \pi \nu}\left(2 \pi \nu \sin 2 \pi \delta_{k}+2 \pi \delta_{k} \cos 2 \pi \delta_{k}\right)\right] \\
& +4 \pi^{2}\left(\delta_{k}^{2}-\nu^{2}\right)\left[2 \pi \nu-4 \pi^{2}\left(\nu^{2}+\delta_{k}^{2}\right)\right. \\
& \left.\left.\left.+e^{-2 \pi \nu}\left(2 \pi \delta_{k} \sin 2 \pi \delta_{k}-2 \pi \nu \cos 2 \pi \delta_{k}\right)\right]\right)\right\}
\end{aligned}
$$

where $\nu=\beta T$ and $\delta_{k}=\omega_{k} T / 2 \pi$. Here, $\beta$ is the laser linewidth. In summary, the Gaussian approximation allows us to consider the decision variable $Y$ in (2) as a Gaussian random variable with conditional mean $\bar{Y}(\boldsymbol{b})=\bar{X}(\boldsymbol{b})$ in (6) and conditional variance $\sigma_{Y}^{2}(\boldsymbol{b})$ given by

$$
\sigma_{Y}^{2}(\boldsymbol{b})=\sigma_{X}^{2}(\boldsymbol{b})+\sigma_{W_{s}}^{2}(\boldsymbol{b})+\sigma_{W_{d k}}^{2}+\sigma_{N}^{2} .
$$

For a threshold $\alpha$, the conditional bit error probability given bit patterns $\boldsymbol{b}^{0}=\left(b_{0}^{0}, b_{1,-1}, \cdots, b_{M,-1}, b_{1,0}, \cdots, b_{M, 0}\right)$ and $\boldsymbol{b}^{1}=\left(b_{0}^{1}, b_{1,-1}, \cdots, b_{M,-1}, b_{1,0}, \cdots, b_{M, 0}\right)$, where $b_{0}^{0}=$ $\sqrt{r /(1+r)}$ and $b_{0}^{1}=\sqrt{1 /(1+r)}$, is [17]

$$
P_{b}\left(b_{1,-1}, \cdots, b_{M,-1} b_{1,0}, \cdots, b_{M, 0}\right)=\frac{1}{2} P_{0}\left(\boldsymbol{b}^{0}\right)+\frac{1}{2} P_{1}\left(\boldsymbol{b}^{1}\right)
$$

where

$$
P_{0}\left(\boldsymbol{b}^{0}\right)=\frac{1}{2} \operatorname{erfc}\left(\frac{\alpha-\bar{Y}\left(\boldsymbol{b}^{0}\right)}{\sqrt{2} \sigma_{Y}\left(\boldsymbol{b}^{0}\right)}\right)
$$

$$
P_{1}\left(\boldsymbol{b}^{1}\right)=\frac{1}{2} \operatorname{erfc}\left(\frac{\bar{Y}\left(\boldsymbol{b}^{1}\right)-\alpha}{\sqrt{2} \sigma_{Y}\left(\boldsymbol{b}^{1}\right)}\right)
$$

and $\operatorname{erfc}(\cdot)$ is defined as

$$
\operatorname{erfc}(a)=\frac{2}{\sqrt{\pi}} \int_{a}^{\infty} e^{-x^{2}} d x
$$

The bit error probability $P_{b}$ is obtained by taking the expectation of $P_{b}\left(b_{1,-1}, \cdots, b_{M,-1}, b_{1,0}, \cdots, b_{M, 0}\right)$ with respect to the bit patterns $\left(b_{1,-1}, \cdots, b_{M,-1}, b_{1,0}, \cdots, b_{M, 0}\right)$. Since there are $2^{2 M}$ such patterns, we have

$$
\begin{aligned}
& P_{b}=\frac{1}{2^{2 M}} \sum_{\left(b_{1,-1}, \cdots, b_{M,-1}, b_{1,0}, \cdots, b_{M, 0}\right)} \\
& \cdot P_{b}\left(b_{1,-1}, \cdots, b_{M,-1}, b_{1,0}, \cdots, b_{M, 0}\right)
\end{aligned}
$$

where the summation is over all $2^{2 M}$ patterns $\left(b_{1,-1}, \cdots, b_{M,-1}, b_{1,0}, \cdots, b_{M, 0}\right)$. The optimal threshold that minimizes the bit error probability is the value that satisfies the following equation:

$$
\begin{array}{r}
\sum_{\left(b_{1,-1}, \cdots, b_{M},-1, b_{1,0}, \cdots, b_{M, 0}\right)}\left(\frac{1}{\sigma_{Y}\left(\boldsymbol{b}^{0}\right)} e^{-\left[\alpha-\bar{Y}\left(\boldsymbol{b}^{0}\right)\right]^{2} / 2 \sigma_{Y}^{2}\left(\boldsymbol{b}^{\mathbf{0}}\right)}\right. \\
\left.-\frac{1}{\sigma_{Y}\left(\boldsymbol{b}^{1}\right)} e^{-\left[\bar{Y}\left(\boldsymbol{b}^{1}\right)-\alpha\right]^{2} / 2 \sigma_{Y}^{2}\left(\boldsymbol{b}^{1}\right)}\right)=0
\end{array}
$$

which is obtained by setting $\partial P_{b} / \partial \alpha$ to zero.

In the case when all laser sources have the same mean wavelength but are uncorrelated, the above results apply by setting $\delta_{k}=0, k=1,2, \cdots, M$ in (8). Furthermore, when all channels are identical, that is, having the same wavelength and phase noise process with possibly the exception of the random initial phases, then $P_{b}$ can be calculated exactly as follows:

$$
P_{b}=E\left\{\frac{1}{4} \operatorname{erfc}\left(\frac{\alpha-x_{0}}{\sqrt{2} \sigma_{0}}\right)+\frac{1}{4} \operatorname{erfc}\left(\frac{x_{1}-\alpha}{\sqrt{2} \sigma_{1}}\right)\right\}
$$

where $x_{0}$ and $x_{1}$ are given in (2b) for logicals zero and one, respectively, and $\sigma_{i}^{2}=\sigma_{N}^{2}+\sigma_{W_{s}}^{2}\left(b^{i}\right)+\sigma_{W_{d k}}^{2}$ with $i=0$ for logical zero and $i=1$ for logical one. The expectation in (12) is carried out over all $\phi_{k}, \tau_{k}$, and bit patterns $\left(b_{1,-1}, \cdots, b_{M,-1}, b_{1,0}, \cdots, b_{M, 0}\right)$. In practice, an upper bound for $P_{b}$ can be obtained without extensive computing by setting $\tau_{k}=0$ for all $k$ (synchronous transmission).

\section{NUMERICAL RESULTS}

In this section, numerical results are presented for a system with a bit rate $500 \mathrm{Mb} / \mathrm{s}$. The responsibility of the photodiode is taken to be 0.5 and the laser extinction ratio is $1 / 20$. The dark current $I_{d k}=10 \mathrm{nA}$. We assume a low noise amplifierintegrate and dump-slicer receiver with an effective noise temperature of $18 \mathrm{~K}$. Assuming a matched load $R_{L}=50$ $\Omega$, the postdetection thermal noise current spectral density is $N_{0}=2 k T_{0} / R_{L}=10^{-23} \mathrm{~A}^{2} / H z$ [13], [20], where $k=$ $1.38 \times 10^{-23} \mathrm{~J} / \mathrm{K}$ is the Boltzmann's constant. The results for both asynchronous (solid line) and synchronous (dashed line) transmissions are plotted in Figs. 2-5 for two adjacent 


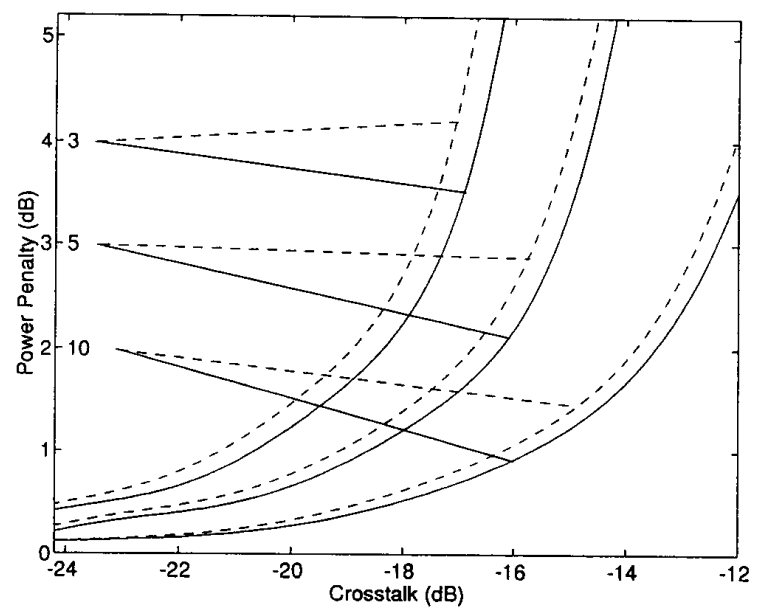

Fig. 2. Power penalty versus crosstalk as a function of normalized laser linewidth for $\delta_{1}=\delta_{2}=0$ (asynchronous: - ; synchronous: $\longrightarrow$ ).

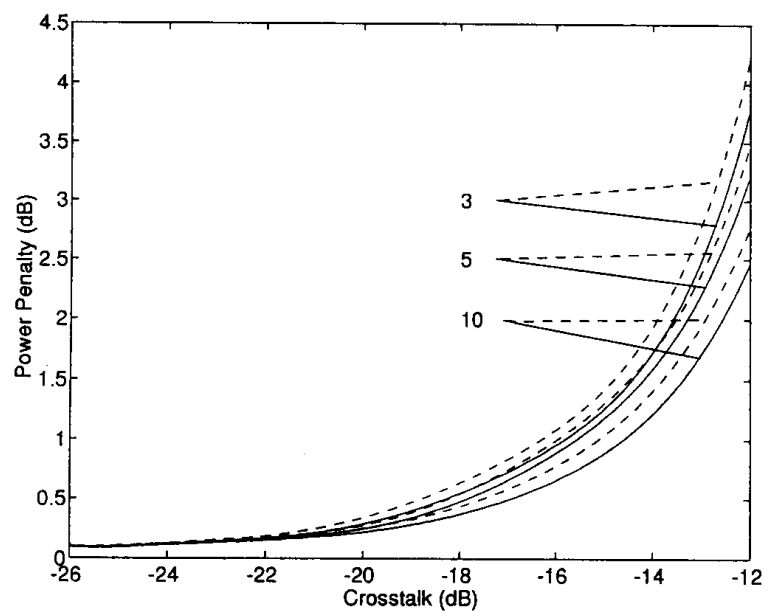

Fig. 3. Power penalty versus crosstalk as a function of normalized laser linewidth for $\delta_{1}=\delta_{2}=5$ (asynchronous: -; synchronous: $\longrightarrow$ ).

channels. It is observed that asynchronous systems perform better than synchronous systems.

Fig. 2 shows the power penalty versus crosstalk as a function of the laser linewidth-bit rate ratio $\nu$ (normalized linewidth) assuming the frequency spacing-bit rate ratios (normalized spacing) to be $\delta_{1}=\delta_{2}=0$. For $1 \mathrm{~dB}$ power penalty, the required crosstalk is $-20.5,-18.5$, and -15.5 $\mathrm{dB}$ for $\nu=3,5$, and 10 , respectively, for asynchronous transmission. When the normalized spacing is increased to five times the bit rate, $\delta_{1}=\delta_{2}=5$, the crosstalk is reduced to about $-16 \mathrm{~dB}$ for $\nu=3$ and $\nu=5$, and $-14.5 \mathrm{~dB}$ for $\nu=$ 10 as shown in Fig. 3. It is seen that both large normalized linewidth and spacing help reduce substantially the crosstalk requirement.

Fig. 4 shows the power penalty versus normalized linewidth $\nu$ as a function of crosstalk for $\delta_{1}=\delta_{2}=0$. For $0.75 \mathrm{~dB}$ power penalty or less, the maximum crosstalk is $-23 \mathrm{~dB}$ for $\nu \geq 2$ and $-20 \mathrm{~dB}$ for $\nu \geq 4$ for asynchronous transmission. Fig. 5 shows the power penalty versus normalized spacing as

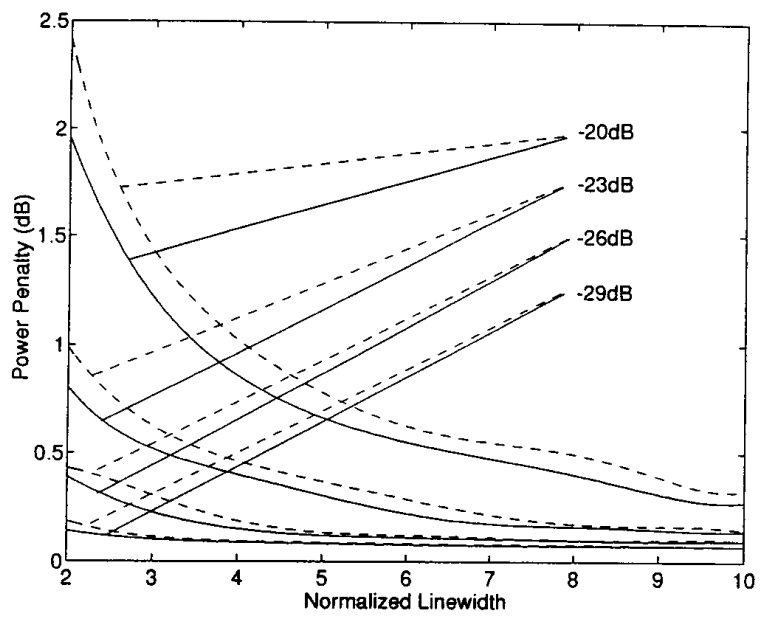

Fig. 4. Power penalty versus normalized laser linewidth as a function of crosstalk for $\delta_{1}=\delta_{2}=0$ (asynchronous: - ; synchronous: - ).

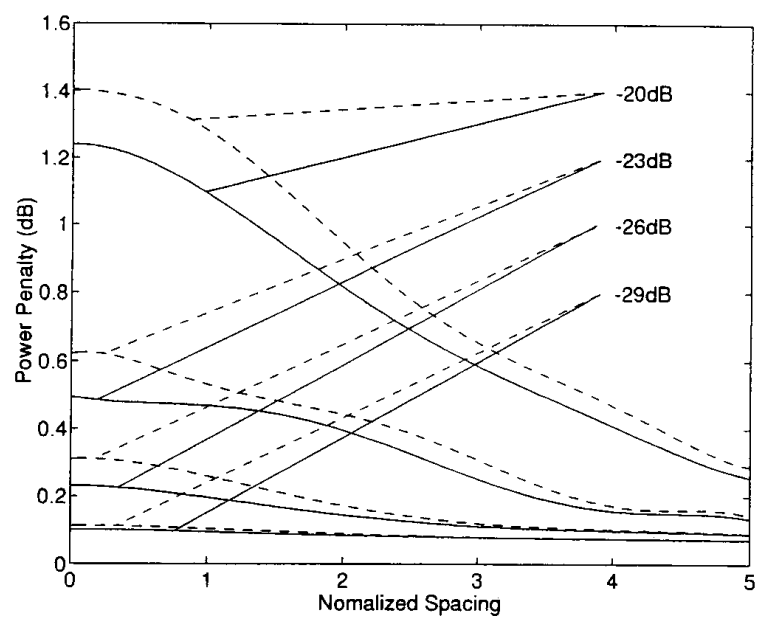

Fig. 5. Power penalty versus normalized frequency spacing as a function of crosstalk for $\nu=3$ (asunchronous: -; synchronous: -

a function of crosstalk for $\nu=3$. For $1 \mathrm{~dB}$ power penalty or less, the maximum crosstalk is $-20 \mathrm{~dB}$ for $\delta_{1}=\delta_{2}>1.3$ for asynchronous transmission.

Fig. 6 shows the power penalty versus crosstalk for synchronous transmission for the case when all channels share the same master source, with the possible exception of the initial phases. This also serves as an upper bound for the asynchronous transmission case whose result is not shown due to prohibitive computer time. For $1 \mathrm{~dB}$ power penalty or less, the maximum crosstalk is about $-25.5 \mathrm{~dB}$. Comparing this to the results when the laser sources are not correlated, it is seen that a much stricter requirement for crosstalk is needed when the laser sources are correlated.

\section{CONCLUSION}

We have presented a mathematical framework to analyze the performance of asynchronous optical chip interconnects in terms of the bit error probability versus the received 


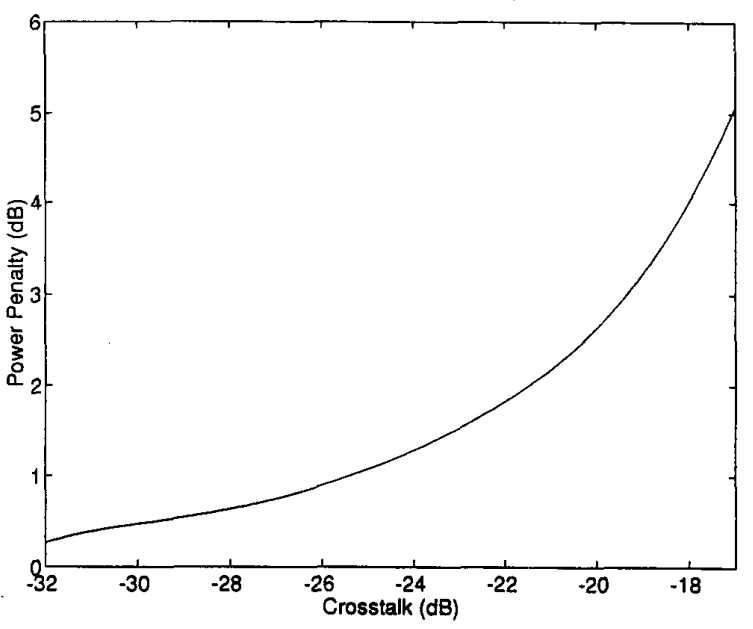

Fig. 6. Power penalty versus crosstalk for the case when all laser sources are correlated (locked to a master source) with the possible exception of the initial phases for synchronous transmission (upper bound for asynchronous transmission)

peak power or power penalty as a function of the crosstalk level, frequency spacing, and laser linewidth. Our analysis can handle any number of adjacent channels. The conclusion drawn from this investigation is that adjacent channels must use laser sources of different wavelengths to reduce the effect of crosstalk. Laser sources with a large linewidth also help, as long as the waveguide bandwidth is much larger than the resulting signal spectrum. In fact, this is the only way to reduce the effect of a given crosstalk when all laser sources have the same mean wavelength. When all laser sources are locked to a master source, the performance depends explicitly on a crosstalk level given a received peak power. Similar conclusions on synchronous transmission appear in [8] via a different mathematical approach that applies to two adjacent channels only. Another conclusion drawn from this study is that asynchronous systems seem to perform better than synchronous systems in all cases.

APPENDIX

DERIVATION OF THE CONDITIONAL Mean SQuare Value of $X$ Given $b$

Let $\boldsymbol{\tau}=\left(\tau_{1}, \tau_{2}, \cdots, \tau_{M}\right)$; we have

$$
\begin{aligned}
E\left\{X^{2} \mid \boldsymbol{b}, \tau\right\}= & \frac{A^{4}}{4} E\left\{T b_{0}^{2}+\sum_{k=1}^{M} C_{k} b_{0} b_{k,-1}\right. \\
& \cdot\left[\int_{0}^{T} e^{j\left[\theta_{0}(t)-\theta_{k}(t)-\omega_{k} t-\phi_{k},-1\right]}\right. \\
& \cdot\left[u(t)-u\left(t-\tau_{k}\right)\right] d t+ \\
& \cdot \int_{0}^{T} e^{-j\left[\theta_{0}(t)-\theta_{k}(t)-\omega_{k} t-\phi_{k,-1}\right]} \\
& \left.\cdot\left[u(t)-u\left(t-\tau_{k}\right)\right] d t\right]
\end{aligned}
$$

$$
\begin{aligned}
& +\sum_{k=1}^{M} C_{k} b_{0} b_{k, 0}\left[\int_{0}^{T} e^{j\left[\theta_{0}(t)-\theta_{k}(t)-\omega_{k} t-\phi_{k, 0}\right]}\right. \\
& \cdot\left[u\left(t-\tau_{k}\right)-u(t-T)\right] d t \\
& +\int_{0}^{T} e^{-j\left[\theta_{0}(t)-\theta_{k}(t)-\omega_{k} t-\phi_{k}, 0\right]} \\
& \left.\cdot\left[u\left(t-\tau_{k}\right)-u(t-T)\right] d t\right] \\
& +\sum_{k=1}^{M} \sum_{l=1}^{M} C_{k} C_{l} b_{k,-1} b_{l,-1} \int_{0}^{T} \\
& \cdot e^{j\left[\theta_{k}(t)-\theta_{l}(t)+\left(\omega_{k}-\omega_{l}\right) t+\phi_{k,-1}-\phi_{l,-1}\right]} \\
& \cdot\left[u(t)-u\left(t-\tau_{k}\right)\right]\left[u(t)-u\left(t-\tau_{l}\right)\right] d t \\
& +\sum_{k=1}^{M} \sum_{l=1}^{M} C_{k} C_{l} b_{k,-1} b_{l, 0} \int_{0}^{T} \\
& \text {. } e^{j\left[\theta_{k}(t)-\theta_{l}(t)+\left(\omega_{k}-\omega_{l}\right) t+\phi_{k,-1}-\phi_{l, 0}\right]} \\
& \cdot\left[u(t)-u\left(t-\tau_{k}\right)\right] \\
& \cdot\left[u\left(t-\tau_{l}\right)-u(t-T)\right] d t \\
& +\sum_{k=1}^{M} \sum_{l=1}^{M} C_{k} C_{l} b_{k,-1} b_{l, 0} \int_{0}^{T} \\
& \text {. } e^{-j\left[\theta_{k}(t)-\theta_{l}(t)+\left(\omega_{k}-\omega_{l}\right) t+\phi_{k},-1-\phi_{l, 0}\right]} \\
& \text { - }\left[u(t)-u\left(t-\tau_{k}\right)\right] \\
& \cdot\left[u\left(t-\tau_{l}\right)-u(t-T)\right] d t \\
& +\sum_{k=1}^{M} \sum_{l=1}^{M} C_{k} C_{l} b_{k, 0} b_{l, 0} \int_{0}^{T} \\
& \text {. } e^{j\left[\theta_{k}(t)-\theta_{l}(t)+\left(\omega_{k}-\omega_{l}\right) t+\phi_{k, 0}-\phi_{l, 0}\right]} \\
& \cdot\left[u\left(t-\tau_{k}\right)-u(t-T)\right] \\
& \left.\cdot\left[u\left(t-\tau_{l}\right)-u(t-T)\right] d t\right\}^{2} \text {. }
\end{aligned}
$$

Expanding the terms, we have

$$
\begin{aligned}
E\left\{X^{2} \mid \boldsymbol{b}, \tau\right\}= & \frac{A^{4}}{4} E\left\{T^{2} b_{0}^{4}+\sum_{k=1}^{M} C_{k}^{2} b_{0}^{2} b_{k,-1}^{2} T \tau_{k}\right. \\
& +\sum_{k=1}^{M} C_{k}^{2} b_{0}^{2} b_{k, 0}^{2} T\left(T-\tau_{k}\right) \\
& +\sum_{k=1}^{M} C_{k}^{2} b_{0}^{2} b_{k,-1}^{2}\left[\int_{0}^{\tau_{k}} \int_{0}^{\tau_{k}} e^{-j \omega_{k}(t-\tau)}\right. \\
& \cdot e^{j\left[\theta_{0}(t)-\theta_{k}(t)-\theta_{0}(\tau)+\theta_{k}(\tau)\right]} d t d \tau \\
& +\int_{0}^{\tau_{k}} \int_{0}^{\tau_{k}} e^{j \omega_{k}(t-\tau)} \\
& \left.\cdot e^{-j\left[\theta_{0}(t)-\theta_{k}(t)-\theta_{0}(\tau)+\theta_{k}(\tau)\right]} d t d \tau\right] \\
& +\sum_{k=1}^{M} C_{k}^{2} b_{0}^{2} b_{k, 0}^{2}\left[\int_{\tau_{k}}^{T} \int_{\tau_{k}}^{T} e^{-j \omega_{k}(t-\tau)}\right. \\
& \cdot e^{j\left[\theta_{0}(t)-\theta_{k}(t)-\theta_{0}(\tau)+\theta_{k}(\tau)\right]} d t d \tau \\
& +\int_{\tau_{k}}^{T} \int_{\tau_{k}}^{T} e^{j \omega_{k}(t-\tau)}
\end{aligned}
$$




$$
\begin{aligned}
& \left.\cdot e^{-j\left[\theta_{0}(t)-\theta_{k}(t)-\theta_{0}(\tau)+\theta_{k}(\tau)\right]} d t d \tau\right] \\
& +\sum_{k=1}^{M} C_{k}^{2} b_{0}^{2} b_{k,-1}^{2} T \tau_{k} \\
& +\sum_{k=1}^{M} \sum_{l=1}^{M} C_{k}^{2} C_{l}^{2} b_{k,-1}^{2} b_{l,-1}^{2} \tau_{k} \tau_{l} \\
& +\sum_{k=1}^{M} \sum_{l=1}^{M} C_{k}^{2} C_{l}^{2} b_{k,-1}^{2} b_{l, 0}^{2} \tau_{k}\left(T-\tau_{l}\right) \\
& +\sum_{k=1}^{M} C_{k}^{2} b_{0}^{2} b_{k, 0}^{2} T\left(T-\tau_{k}\right) \\
& +\sum_{k=1}^{M} \sum_{l=1}^{M} C_{k}^{2} C_{l}^{2} b_{k,-1}^{2} b_{l, 0}^{2} \tau_{k}\left(T-\tau_{l}\right) \\
& \left.+\sum_{k=1}^{M} \sum_{l=1}^{M} C_{k}^{2} C_{l}^{2} b_{k, 0}^{2} b_{l, 0}^{2}\left(T-\tau_{k}\right)\left(T-\tau_{l}\right)\right\}
\end{aligned}
$$

Evaluating the expection of each term, we get

$$
\begin{aligned}
E\left\{X^{2} \mid \boldsymbol{b}, \tau\right\}= & \frac{A^{4}}{4}\left\{T^{2} b_{0}^{4}+2 \sum_{k=1}^{M} C_{k}^{2} b_{0}^{2} b_{k,-1}^{2} T \tau_{k}\right. \\
& +2 \sum_{k=1}^{M} C_{k}^{2} b_{0}^{2} b_{k, 0}^{2} T\left(T-\tau_{k}\right) \\
& +2 \sum_{k=1}^{M} \sum_{l=1}^{M} C_{k}^{2} C_{l}^{2} b_{k,-1}^{2} b_{l, 0}^{2} \tau_{k}\left(T-\tau_{l}\right) \\
& +\sum_{k=1}^{M} \sum_{l=1}^{M} C_{k}^{2} C_{l}^{2} b_{k,-1}^{2} b_{l,-1}^{2} \tau_{k} \tau_{l} \\
& +\sum_{k=1}^{M} \sum_{l=1}^{M} C_{k}^{2} C_{l}^{2} b_{k, 0}^{2} b_{l, 0}^{2}\left(T-\tau_{k}\right)\left(T-\tau_{l}\right) \\
& +\sum_{k=1}^{M} C_{k}^{2} b_{0}^{2} b_{k,-1}^{2}\left[\int_{0}^{\tau_{k}} \int_{0}^{\tau_{k}} e^{-j \omega_{k}(t-\tau)}\right. \\
& \cdot E\left\{e^{j\left[\theta_{0}(t)-\theta_{k}(t)-\theta_{0}(\tau)+\theta_{k}(\tau)\right]}\right\} d t d \tau \\
& +\int_{0}^{\tau_{k}} \int_{0}^{\tau_{k}} e^{j \omega_{k}(t-\tau)} \\
& \left.\cdot E\left\{e^{-j\left[\theta_{0}(t)-\theta_{k}(t)-\theta_{0}(\tau)+\theta_{k}(\tau)\right\}}\right\} d t d \tau\right] \\
& \cdot \sum_{k=1}^{M} C_{k}^{2} b_{0}^{2} b_{k, 0}^{2}\left[\int_{\tau_{k}}^{T} \int_{\tau_{k}}^{T} e^{-j \omega_{k}(t-\tau)}\right. \\
& \cdot E\left\{e^{j\left[\theta_{0}(t)-\theta_{k}(t)-\theta_{0}(\tau)+\theta_{k}(\tau)\right]}\right\} d t d \tau \\
& +\int_{\tau_{k}}^{T} \int_{\tau_{k}}^{T} e^{j \omega_{k}(t-\tau)} \\
& \left.\left.E\left\{e^{-j\left[\theta_{0}(t)-\theta_{k}(t)-\theta_{0}(t)+\theta_{k}(\tau)\right]}\right\} d t d \tau\right]\right\} \\
& \\
& \\
&
\end{aligned}
$$

The conditional variance of $X$ given by $\boldsymbol{b}$ and $\boldsymbol{\tau}$ is given by

$$
\begin{aligned}
\sigma_{X}^{2}(\boldsymbol{b}, \boldsymbol{\tau})= & E\left\{X^{2} \mid \boldsymbol{b}, \boldsymbol{\tau}\right\}-[\bar{X}(\boldsymbol{b}, \boldsymbol{\tau})]^{2} \\
= & \frac{A^{4}}{4}\left\{\sum _ { k = 1 } ^ { M } C _ { k } ^ { 2 } b _ { 0 } ^ { 2 } b _ { k , - 1 } ^ { 2 } \left[\int_{0}^{\tau_{k}} \int_{0}^{\tau_{k}} e^{-j \omega_{k}(t-\tau)}\right.\right. \\
& \cdot E\left\{e^{j\left[\theta_{0}(t)-\theta_{k}(t)-\theta_{0}(\tau)+\theta_{k}(\tau)\right]}\right\} d t d \tau \\
& +\int_{0}^{\tau_{k}} \int_{0}^{\tau_{k}} e^{j \omega_{k}(t-\tau)} \\
& \left.\cdot E\left\{e^{-j\left[\theta_{0}(t)-\theta_{k}(t)-\theta_{0}(\tau)+\theta_{k}(\tau)\right]}\right\} d t d \tau\right] \\
& \cdot \sum_{k=1}^{M} C_{k}^{2} b_{0}^{2} b_{k, 0}^{2}\left[\int_{\tau_{k}}^{T} \int_{\tau_{k}}^{T} e^{-j \omega_{k}(t-\tau)}\right. \\
& \cdot E\left\{e^{j\left[\theta_{0}(t)-\theta_{k}(t)-\theta_{0}(\tau)+\theta_{k}(\tau)\right]}\right\} d t d \tau \\
& +\int_{\tau_{k}}^{T} \int_{\tau_{k}}^{T} e^{j \omega_{k}(t-\tau)} \\
& \left.\left.\cdot E\left\{e^{-j\left[\theta_{0}(t)-\theta_{k}(t)-\theta_{0}(\tau)+\theta_{k}(\tau)\right]}\right\} d t d \tau\right]\right\}(\mathrm{A}
\end{aligned}
$$

In the derivations of (A4), we have used the fact that all $\phi_{k}, k, 1,2, \cdots, M$ are uniform random variables over $(0,2 \pi)$.

Let $\psi=\theta_{0}(t)-\theta_{k}(t)-\left[\theta_{0}(\tau)-\theta_{k}(\tau)\right]$. The laser phase noise $\theta_{k}(t), k=0,1, \cdots, M$ is characterized by a Wiener process [17], [18] such that $d \theta_{k}(t) / d t=2 \pi \mu(t)$, where $\mu(t)$ is a zero mean white Gaussian process of $\operatorname{PSD} \beta / 2 \pi \mathrm{Hz}$, where $\beta$ is the laser linewidth assumed to be the same for all lasers sources. The variance of $\theta_{k}(t)$ is $2 \pi \beta t$. The process $\psi$ is a zero mean Gaussian process and can be expressed as

$$
\begin{aligned}
\psi= & 2 \pi \int_{0}^{t}\left[\mu_{0}\left(t_{1}\right)-\mu_{k}\left(t_{1}\right)\right] d t_{1} \\
& -2 \pi \int_{0}^{\tau}\left[\mu_{0}\left(t_{1}\right)-\mu_{k}\left(t_{1}\right)\right] d t_{1} \\
& =2 \pi \int_{\tau}^{t}\left[\mu_{0}\left(t_{1}\right)-\mu_{k}\left(t_{1}\right)\right] d t_{1} .
\end{aligned}
$$

Therefore, the variance $\sigma_{\psi}^{2}$ is given by

$$
\begin{aligned}
\sigma_{\psi}^{2}= & 4 \pi^{2} \int_{\tau}^{t} \int_{\tau}^{t} E\left\{\left[\mu_{0}\left(t_{1}\right)-\mu_{k}\left(t_{1}\right)\right]\right. \\
& \left.\cdot\left[\mu_{0}\left(t_{2}\right)-\mu_{k}\left(t_{2}\right)\right]\right\} d t_{1} d t_{2} \\
& =4 \pi^{2} \int_{\tau}^{t} \int_{\tau}^{t} \frac{\beta}{\pi} \delta\left(t_{1}-t_{2}\right) d t_{1} d t_{2} \\
& =4 \pi \beta \int_{-|t-\tau|}^{|t-\tau|}(|t-\tau|-|u|) \delta(u) d u \\
& =4 \pi \beta|t-\tau| .
\end{aligned}
$$

Using the fact that $\psi$ and $-\psi$ are both Gaussian random variables with zero mean and variance $4 \pi \beta|t-\tau|$, we obtain

$$
E\left\{e^{j \psi}\right\}=E\left\{e^{-j \psi}\right\}=e^{-\sigma_{\psi}^{2} / 2}=e^{-2 \pi \beta|t-\tau|} .
$$


Substituting (A7) into (A4), we obtain

$$
\begin{aligned}
\sigma_{X}^{2}(\boldsymbol{b}, \boldsymbol{\tau})= & \frac{A^{4}}{2}\left\{\sum_{k=1}^{M} C_{k}^{2} b_{0}^{2} b_{k,-1}^{2} \int_{0}^{\tau_{k}} \int_{0}^{\tau_{k}} e^{-2 \pi \beta|t-\tau|}\right. \\
& \cdot \cos \omega_{k}(t-\tau) d t d \tau+\sum_{k=1}^{M} C_{k}^{2} b_{0}^{2} b_{k, 0}^{2} \int_{\tau_{k}}^{T} \int_{\tau_{k}}^{T} \\
& \left.\cdot e^{-2 \pi \beta|t-\tau|} \cos \omega_{k}(t-\tau) d t d \tau\right\} \\
& =\frac{A^{4}}{2}\left\{\sum_{k=1}^{M} C_{k}^{2} b_{0}^{2} b_{k,-1}^{2} \int_{-\tau_{k}}^{\tau_{k}}\left(\tau_{k}-|u|\right) e^{-2 \pi \beta|u|}\right. \\
& \cdot \cos \omega_{k} u d u \\
& +\sum_{k=1}^{M} C_{k}^{2} b_{0}^{2} b_{k, 0}^{2} \int_{-\left|T-\tau_{k}\right|}^{\left|T-\tau_{k}\right|}\left(\left|T-\tau_{k}\right|-|u|\right) \\
& \left.\cdot e^{-2 \pi \beta|u|} \cos \omega_{k} u d u\right\}
\end{aligned}
$$

The conditional variance of $X$ given $b$ is obtained by averaging $\sigma_{X}^{2}(\boldsymbol{b}, \tau)$ over $\tau$

$$
\begin{aligned}
\sigma_{X}^{2}(\boldsymbol{b})= & \sum_{k=1}^{M} C_{k}^{2} A^{4} T^{2} b_{0}^{2}\left(b_{k,-1}^{2}+b_{k, 0}^{2}\right)\left\{\frac{\pi \nu}{4 \pi^{2}\left(\nu^{2}+\delta_{k}^{2}\right)}\right. \\
& -\frac{1}{64 \pi^{6}\left(\nu^{2}+\delta_{k}^{2}\right)^{3}}\left(8 \pi^{2} \nu \delta_{k}\right. \\
& \cdot\left[2 \pi \delta_{k}-e^{-2 \pi \nu}\left(2 \pi \nu \sin 2 \pi \delta_{k}+2 \pi \delta_{k} \cos 2 \pi \delta_{k}\right)\right] \\
& +4 \pi^{2}\left(\delta_{k}^{2}-\nu^{2}\right)\left[2 \pi \nu-4 \pi^{2}\left(\nu^{2}+\delta_{k}^{2}\right)\right. \\
& \left.\left.\left.+e^{-2 \pi \nu}\left(2 \pi \delta_{k} \sin 2 \pi \delta_{k}-2 \pi \nu \cos 2 \pi \delta_{k}\right)\right]\right)\right\}(\text { A.9 })
\end{aligned}
$$

where $\nu=\beta T$ and $\delta_{k}=\omega_{k} T / 2 \pi$.

\section{REFERENCES}

[1] B. L. Booth, "Low loss channel waveguides in polymers," J. Lightwave Technol., vol. LT-7, pp. 1445-1453, Oct. 1989.

[2] J. M. Trewhella et al., "Polymetric optical waveguides," SPIE, vol. 1177, pp. 379-386, 1989.

[3] W. K. Burns, "Normal mode analysis of waveguides devices. Part I: Theory," J. Lightwave Technol., vol. LT-6, pp. 1051-1057, June 1988.

[4] H. A. Hauss, W. P. Huang, S. Kawakami, and N. A. Whitaker, "Coupler mode theory of optical waveguides," J. Lightwave Technol., vol. LT-5, pp. 16-23, Jan. 1987.

[5] S. Kawakami and H. A. Hauss, "Continuum analog of coupled multiple waveguides," J. Lightwave Technol., vol. LT-4, pp. 160-168, Feb, 1986.
[6] A. Hardy and W. Streifer, "Couple modes of multiwaveguides systems and phase arrays," J. Lightwave Technol., vol. LT-4, pp. 90-99, Jan. 1986.

[7] W. Stutius and W. Streifer, "Silicon nitride films on silicon for optical waveguides," Appl. Opt., vol. 6, no. 12, pp. 3218-3222, Dec. 1977.

[8] C. S. Li, C. M. Olsen, and D. G. Messerschmitt, "Analysis of crosstalk penalty in dense optical chip interconnects using single-mode waveguides," J. Lightwave Technol., vol. 9, pp. 1693-1701, Dec. 1991.

[9] M. Dagenais, R. Leheny, H. Temkin, and P. B. Bhattacharya, "Applications and challenges of OEIC technology: A report on the 1989 Hilton Head Workshop," J. Lightwave Technol., vol. 8, pp. 846-862, June 1990.

[10] J. Gowar, Optical Communication Systems. Englewood Cliffs, NJ: Prentice-Hall, 1984.

[11] G. E. Keiser, Optical Fiber Communications, 2nd ed. New York: McGraw-Hill, 1992.

[12] J. P. Powers, An Introduction to Fiber Optics Systems. Aksen Associates, 1993.

[13] A. Yariv, Optical Electronics, 3rd ed. New York: Holt, Rinehart, Winston, 1985.

[14] J. R. Barry and E. A. Lee, "Performance of coherent optical receivers," Proc. IEEE, vol. 78, pp. 1369-1394, Aug. 1990.

[15] L. G. Kazorsky and O. K. Tonguz, "ASK and FSK coherent lightwave systems: A simplified approximate analysis," J. Lightwave Technol., vol. 8, pp. 338-352, Mar. 1990.

[16] L. G. Kazovsky, P. Meissner, and E. Patzak, "ASK multiport optical homodyne receivers," J. Lightwave Technol., vol. LT-5, pp. 770-791, June 1987.

[17] L. W. Couch II, Digital and Analog Communications Systems, 4th ed. New York: MacMillan, 1993.

[18] J. Salz, "Coherent lightwave communications, AT\&T Tech. J., vol. 64 pp. 2153-2209, Dec. 1985

[19] G. J. Foschini, L. J. Greenstein, and G. Vannucci, "Noncoherent detection of coherent lightwave signals corrupted by phase noise," IEEE Trans. Commun., vol. COM-36, pp. 306-314, Mar. 1988.

[20] A. Papoulis, Probability, Random Variables, and Stochastic Processes. New York: McGraw-Hill, 1985.

[21] J. G. Proakis, Digital Communications, 2nd ed. New York: McGrawHill, 1989.

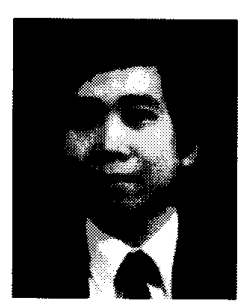

Tri T. Ha (M'78-SM'85-F'93) received the Ph.D degree in electrical engineering from the University of Maryland in 1977. He has worked for Fairchild Industries, GTE, and the Virginia Polytechnic Institute and State University. Since 1987, he has been with the Naval Postgraduate School where he is currently a professor of electrical and computer engineering. He is the author of two books and has authored and co-authored more than 60 technical papers. His present interest is optical networks. Ha is an IEEE Fellow.

Rena M. Loesch, LT, USN, received her B.S. degree in chemistry and mathematics from the University of California at Los Angeles in 1983 and her M.S. degree in electrical engineering from the Naval Postgraduage School in Monterey, California in 1993. Previous tours in the Navy have been at the Navy Regional Data Automation Center in Washington, D.C. and at the David Taylor Research Center in Carderock, Maryland. She is currently stationed at the Navy Communication Detachment in Cheltenham, Maryland. 